\title{
CORRELATION BETWEEN LUNG AND JOINT INVOLVEMENT IN PATIENTS WITH RHEUMATOID ARTHRITIS AND INTERSTITIAL LUNG DISEASE: A CROSS-SECTIONAL STUDY
}

\author{
Francisco Paulin ${ }^{1}$, Juan Francisco Mercado ${ }^{2}$, Martín Eduardo Fernández ${ }^{1}$, Fabián Matías Caro ${ }^{1}$, \\ María Laura Alberti ${ }^{1}$, Leandro Alberto Fassola ${ }^{1}$
}

${ }^{1}$ Interstitial Lung Disease Clinic, María Ferrer Hospital, Buenos Aires, Argentina; ${ }^{2}$ Internal Medicine Department, University of Pittsburgh Medical Center, Pittsburgh, PA, USA

\begin{abstract}
Background: Rheumatoid arthritis (RA) can affect the lungs in different manners, with interstitial lung disease (ILD) as the most serious manifestation. Although lung and joint compromise could be thought to evolve in parallel, there are data suggesting the opposite. In this study, we evaluated the relationship between lung and joint involvement in RA ILD. Methods: An observational cross-sectional study of RA ILD patients evaluated from January 2015 to February 2017. Joint disease assessment included number of tender and swollen joints, patient's global assessment of disease activity, erythrocyte sedimentation rate (ESR) or C-reactive protein, and disease activity score (DAS28). Lung disease assessment included forced vital capacity, diffusion capacity (DLCO), and Goh high-resolution computed tomography (HRCT) score for total extent, ground glass, and reticular pattern. We studied the correlation between both components of the disease. Results: We included 46 patients, 14 (30.4\%) men, with a mean (SD) of the age of 59.9 years (11.89). 12 (26.09) patients were in remission or had low disease activity measured with DAS28. The HRCT showed usual interstitial pneumonia (UIP) pattern in 10 (21.7\%), possible UIP in 18 (39.1\%), and inconsistent with UIP in 18 (39.1\%). We found a good correlation between the ESR and the ground glass score in the HRCT $(r=0.39 ; p=0.03)$. However, we found no correlation between lung function tests or HRCT scores and the other components of the DAS28. Conclusions: We only found a good correlation between ESR and ground glass score. It is possible that different pathways of the immune response mediate damage in lungs and joints. (REV INVES CLIN. 2018;70:76-81)
\end{abstract}

Key words: Rheumatoid arthritis. Interstitial lung disease. Usual interstitial pneumonia. Lung fibrosis.

Corresponding author:

*Francisco Paulin Interstitial Lung Disease Clinic

María Ferrer Hospital

Dr. Enrique Finochietto 849

C.P. C1272AAA, Buenos Aires, Argentina

Email: francisco_paulin@yahoo.com.ar

Received for publication: 29-12-2017

Accepted for publication: 04-02-2018

doi: $10.24875 / R I C .18002474$ 


\section{INTRODUCTION}

Rheumatoid arthritis (RA) is the most common autoimmune disease in adults with a prevalence of approximately $1 \%$ in the general population ${ }^{1}$. It can affect the lungs in different manners, with interstitial lung disease (ILD) as the most serious manifestation, given its impact on prognosis ${ }^{2,3}$. The presence of anticyclic citrullinated protein (ACCP) antibodies, rheumatoid factor (RF), male sex, higher erythrocyte sedimentation rate (ESR), and higher disease activity score (DAS28) values is associated with ILD ${ }^{4}$. Almost all the high-resolution computed tomography (HRCT) patterns observed in the idiopathic ILDs are associated with RA ${ }^{5}$. In this disease spectrum, the usual interstitial pneumonia (UIP) pattern represents the fibrotic pole, and the nonspecific interstitial pneumonia (NSIP) pattern the inflammatory one ${ }^{6}$. This morphological difference has clinical and prognostic implications since the NSIP survival is twice longer than that of UIP ${ }^{7,8}$.

As ILD represents the lung compromise of a systemic disease that mainly affects joints, it could be logical to assume that the disease activity in both sites should have good correlation. However, this fact has not been demonstrated yet. Only one observational study has been conducted to evaluate the relationship between lung and joint involvement in these patients, which showed a positive correlation between the clinical disease activity index (CDAl) and ground-glass scores on $\mathrm{HRCT}^{9}$. However, there are some elements that suggest that this fact should not be considered as a rule. In the first place, although the available evidence is controversial, it has been reported that some agents used successfully to treat the articular disease may not have the same effectiveness, and can even be harmful when they are used to treat RA patients with ILD 10-13. This suggests that the mechanisms that mediate both processes could be quite different. Finally, it has been proposed that a primary fibrotic process could be central for the development of the UIP pattern, resembling the picture of idiopathic pulmonary fibrosis (IPF), independently from the inflammatory phenomenon that takes place in the joints ${ }^{14}$. According to this hypothesis, the group of patients with UIP pattern may have progression of the ILD, which may dissociate from joint disease activity. The presence or absence of correlation between both disease manifestations has therapeutic implications since it could represent the evidence of distinct mechanisms taking place in both sites, with different pathways to target. In the era of the treatments directed against specific targets, this fact becomes relevant. With this background, we decided to study the correlation between the disease activity in lungs and joints in RA ILD patients.

\section{PATIENTS AND METHODS}

We conducted an observational cross-sectional study of RA ILD patients evaluated from January 2015 to February 2017 in the ILD clinic of our hospital. All the patients met ACR 2010 criteria for RA ${ }^{15}$. As our hospital is a respiratory disease referral center, almost all patients were sent by their personal rheumatologist due to the presence of respiratory symptoms. We defined the presence of ILD (based on a compatible HRCT) when a consensus between the immunologist, the radiologist, and the pulmonologist was reached. Patients who had a period longer than 3 months between articular and lung disease evaluations were excluded.

\section{Joint disease assessment}

DAS are validated instruments created to evaluate joint compromise in RA, with cutoff points that discriminate between remission, mild, moderate, and high disease activity. DAS28 is one of the most utilized scores ${ }^{16,17}$. It includes the number of tenders and swollen joints, a visual analog scale that measures patient's global assessment of disease activity, and either ERS or C-reactive protein. We studied the correlation between each of these components and lung disease parameters. The articular evaluation was performed by the same clinical staff in all the patients.

Anti-citrullinated protein antibodies (ACPAs) were measured by ELISA (positivity $>20$ units $/ \mathrm{mL}$ ). RF was measured by immunoturbidimetry (positivity $>20$ international units $/ \mathrm{mL}$ ).

\section{Lung disease assessment}

We evaluated the lung functional status using percent forced vital capacity (FVC) and diffusion capacity (DLCO). Lung functional tests were performed according to international standards ${ }^{18}$. HRCTs were 
performed with slides 1-3 mm thick, and approximately 20 slides per patient. Slides were evaluated by a radiologist with experience in ILD. This reader was blinded to clinical, serological, and functional data. The pattern was classified according to the 2011 ATS/ERS/JRS/ALAT consensus into one of three categories: UIP pattern, possible UIP pattern, and inconsistent with UIP pattern ${ }^{19}$. The scans were scored using the system proposed by Goh et al. ${ }^{20}$ This score contemplates five levels: origin of great vessels, main carina, pulmonary venous confluence, halfway between the third and fifth section, and immediately above the right hemidiaphragm. At every level, total disease extent, and the proportions of reticular and ground-glass opacities were scored. An average between the five mentioned levels was calculated for each component of the score (total extent, ground glass, and reticulation). We evaluated the intraclass correlation coefficient (ICC) of our radiologist. The ICC of our reader was 0.93 (95\% confidence interval [Cl], 0.80-0.97; $\mathrm{p}<0.001$ ) for the total disease extent score, $0.93(95 \% \mathrm{Cl}, 0.82-0.97 ; \mathrm{p}<0.001)$ for the ground glass score, and $0.95(95 \% \mathrm{Cl}, 0.89-0.98$; $\mathrm{p}<0.001$ ) for the reticular score. The correlation of total disease extent and FVC $\%$ was $-0.58(p=0.009)$.

\section{Statistical analysis and power calculation}

Correlation analysis was performed using Spearman or Pearson's methods depending on the presence of normal distribution. Significance was defined as a $p=0.05$ or lower. For an expected $r=0.4$, we calculated a power of 0.72 for the correlation analysis between HRCT scores and joint involvement, and a power of 0.80 for the correlation analysis between lung function tests and joint disease.

The manuscript was written according to the STROBE initiative for the communication of observational studies $^{21}$. The Institutional Ethics Committee of our hospital reviewed and approved the study. Patients gave their informed consent before their inclusion into the study.

\section{RESULTS}

In the period from January 2015 to February 2017, 60 patients with RA and lung compromise were
Figure 1. Scatter plot between ESR and ground glass Goh score, $R=0.39(p=0.03)$.

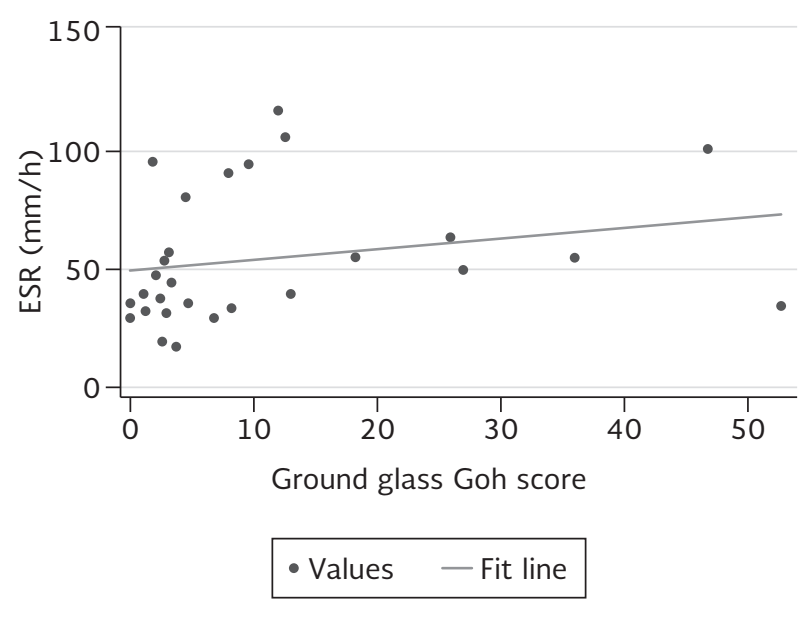

evaluated at the ILD clinic of our hospital. Of these, 46 had a complete articular evaluation, including DAS28 score and at least one of the two methods used to assess lung compromise (HRCT or lung function test), with $<3$ months between them. All patients performed lung function tests. In 40 patients, the specific CT slides proposed by Goh et al. were available to analyze disease extent scores. From the 46 patients, the mean (SD) of age was 59.9 years (11.89) when the ILD was diagnosed, and 14 (30.4\%) were men. The median of time (IQR) between the diagnosis of RA and the diagnosis of ILD was 40 months (12-100). 31 patients (67.4\%) were current or past smokers. Only two patients had lung biopsy performed to make the diagnosis of the ILD pattern. We found evidence of an inflammatory pattern in both biopsies, specifically a NSIP pattern. Regarding the autoantibodies status, $92.5 \%$ were ACPA positive (37/40), and $97.3 \%$ were positive for RF (36/37). 12 (29\%) patients were in remission or had low disease activity measured by DAS28. The HRCT showed a UIP pattern in $10(21.7 \%)$, possible UIP in $18(39.1 \%)$, and inconsistent with UIP in 18 (39.1\%). Characteristics of the cohort related to the lung and joint disease activity are shown in table 1 .

Regarding the correlation analysis, we found a good correlation between the ESR and the ground glass score in the HRCT $(r=0.39 ; p=0.03)$ (Fig. 1). We excluded other causes of elevated ESR such as infection. Of the patients included for this analysis, 19 (65\%) were receiving methotrexate, 16 (55\%) leflunomide, $5(17 \%)$ an anti-TNF agent, and $3(10 \%)$ were 
Table 1. Characteristics of patients $(n=46)$ with rheumatoid arthritis and interstitial lung disease

\begin{tabular}{ll}
\hline $\begin{array}{l}\text { Dyspnea duration, months, } \\
\text { median (IQR) }\end{array}$ & $6(3-18)$ \\
UIP pattern, $\mathrm{n}(\%)$ & $10(21.7)$ \\
Possible UIP pattern, n (\%) & $18(39.1)$ \\
Inconsistent with UIP, n (\%) & $18(39.1)$ \\
*Goh total extent, median (IQR) & $22(13-36)$ \\
Goh ground glass, median (IQR) & $3.7(2.1-12.5)$ \\
Goh reticulation, median (IQR) & $14.4(8.6-24)$ \\
FVC\%, mean (SD) & $71(17.48)$ \\
TLC\%, mean (SD) & $76.29(15.29)$ \\
DLCO\%, mean (SD) & $59.02(22.91)$ \\
ESR, mean (SD) & $51.9(28.56)$ \\
DAS28, mean (SD) & $4.43(1.48)$ \\
DAS28 remission or low activity, & $12(26.09)$ \\
$\mathrm{n}(\%)$ & $2(0-6)$ \\
Tender joints, median (IQR) & $2(1-5)$ \\
Swelling joints, median (IQR) & $43.9(31.57)$ \\
PGA of disease activity, & \\
median (IQR) & $36 / 37(97.3)$ \\
RF, n (\%) & $37 / 40(92.5)$ \\
ACCP, n (\%) & $28(60.87)$ \\
Methotrexate, n (\%) & $21(48.84)$ \\
Leflunomide, $\mathrm{n}(\%)$ & \\
Anti-TNF, n (\%) & \\
Other biological agents, n (\%) & \\
\hline
\end{tabular}

*Goh staging system (20).

UIP: usual interstitial pneumonia; FVC: forced vital capacity;

TLC: total lung capacity; DLCO: diffusion capacity; ERS: erythrocyte sedimentation rate; DAS 28: disease activity score 28 ; PGA: patient global assessment; RF: rheumatoid factor; ACCP: anti-citrullinated antibodies.

receiving other biological agents. We did not find a correlation between lung function tests (FVC \% or DLCO \%) and joint involvement parameters. We also found no correlation between the joint compromise parameters and the other components of the Goh score in HRCT (total extent and reticular opacities). The complete correlation analysis is shown in table 2 .

\section{DISCUSSION}

In this study, we present a cohort of RA ILD patients, with a predominantly fibrotic lung compromise, and an articular disease activity from moderate to high. In our opinion, these results are not surprising, because previous studies have already demonstrated that patients with RA ILD have high scores of disease activity $^{7}$. Furthermore, most of the patients resulted positive to anti-CCP antibodies and RF. Almost all the patients included had RA diagnosis before ILD diagnosis. As a consequence, the vast majority were receiving treatment with drugs that can eventually affect the lungs. Our results show a good correlation between ESR and the ground glass score on HRCT in patients with RA ILD. We found no correlation between lung compromise (evaluated with lung function tests and HRCT scores), and joint involvement evaluated with DAS28. In our opinion, these results are interesting and warrant an extensive debate related to the different manifestations of this disease, and the pathways involved in them. The prevalence of ILD in RA patients seems to be greater than it was traditionally thought. Some studies have found a prevalence of $33-42 \%$ of interstitial abnormalities when patients are screened using HRCT 22,23 . This is remarkable because ILD is an important cause of death in RA patients. ILD can precede the onset of articular involvement in some patients ${ }^{24,25}$. In recent years, an important number of agents have been approved to be used in RA patients ${ }^{17}$. However, no clinical trials have been carried out to find new treatments for the

Table 2. Correlation analysis between lung and joint disease parameters in patients with rheumatoid arthritis and interstitial lung disease

\begin{tabular}{|c|c|c|c|c|c|}
\hline & DAS 28 & Joint swelling & Joint tenderness & $\begin{array}{c}\text { Disease activity } \\
\text { PGA }\end{array}$ & ESR \\
\hline FVC\% & $0.16(0.32)$ & $0.006(0.96)$ & $0.15(0.35)$ & $-0.16(0.27)$ & $-0.10(0.59)$ \\
\hline DLCO\% & $-0.02(0.88)$ & $-0.05(0.74)$ & $0.06(0.69)$ & $-0.17(0.26)$ & $-0.14(0.45)$ \\
\hline Goh score total extent & $-0.07(0.65)$ & $-0.15(0.35)$ & $-0.01(0.93)$ & $0.06(0.71)$ & $0.22(0.25)$ \\
\hline Goh score ground glass & $0.01(0.92)$ & $0.02(0.89)$ & $0.05(0.74)$ & $0.17(0.29)$ & $0.39(0.03)$ \\
\hline Goh score reticulation & $-0.22(0.194)$ & $-0.28(0.081)$ & $-0.29(0,078)$ & $-0.16(0.31)$ & $-0.03(0.85)$ \\
\hline
\end{tabular}

Results expressed as correlation coefficients ( $p$ value).

DAS28: disease activity score 28; PGA: patient global assessment; ERS: erythrocyte sedimentation rate; FVC: forced vital capacity;

DLCO: diffusion capacity. 
lung component of the disease. It could be logical to assume that agents used successfully to treat the articular component of the disease are also useful to treat ILD. Even so, this fact does not appear to be true. Although the available evidence is controversial, an increasing number of studies have appeared in the last years reporting the deterioration of a pre-existing ILD in RA patients receiving anti-TNF agents ${ }^{10-13,26}$. This fact is certainly surprising, since anti-TNF agents have proven to be an effective alternative to treat patients with arthritis refractory to synthetic diseasemodifying antirheumatic drugs (e.g., methotrexate or leflunomide $)^{17}$. A possible explanation is that the mechanisms behind lung and joint injury in RA are distinct. Supporting this hypothesis, the inflammatory infiltrates found on both sites are quite different. Lung tissue infiltrates show lymphoid aggregates and even lymphoid follicles with germinal centers ${ }^{27,28}$. On occasions, plasma cells and dendritic cells are also present. On the other hand, macrophages, neutrophils, and fibroblast-like synoviocytes are the dominant cellular populations in the synovial tissue ${ }^{29}$. This picture could reveal that distinct pathways of the immune response are mediating the damage in lungs and joints, and thus explain why immunosuppressant agents have different effects in both sites. Furthermore, it could also explain the absence of correlation between lung and joint disease we found in our study.

It is remarkable that in our study, ESR showed a good correlation with the ground glass score in HRCT. This reflects the inflammatory nature of the ground glass opacities, a fact that is assumed but has not been demonstrated before in this group of patients. Our finding has therapeutic implications, because currently, we can choose between different strategies to treat patients with ILD (antifibrotics and/or immunosuppressive agents). In recent years, new theories have been proposed to explain the pathophysiology of RA ILD ${ }^{14,30,31}$. Some of them propose that, in patients with UIP pattern, the alveolar epithelial cell directs the fibrotic process (a process that is independent of the immune response), resembling what occurs in IPF. Subsequently, peptides are citrullinated in the lung parenchyma; an immune response against them develops, and citrullinated peptides are targeted both in lungs and joints. In this scenario, the lung and joint components of the disease can have different behaviors. This hypothesis could help explain our results since the dissociation of the disease activity in both sites can be related to the predominantly fibrotic phenotype of the ILD which we found in our cohort. In agreement with these arguments, another group of researchers found that lower fibrotic scores predicted response to immunosuppressive treatment in RA ILD patients ${ }^{32}$. There is only one retrospective study before ours evaluating the correlation between lung and joint disease 9 . The authors included 34 patients and found a good correlation $(r=0.37, p=0.028)$ between HRCT ground glass scores and joint disease activity. There are some differences with our study. First, they used a different HRCT score, the one proposed by Kazeroni et al., ${ }^{33}$ and a different disease activity index (clinical disease activity index or CDAI). We preferred Goh score because it was developed and validated to be utilized in patients with ILD related to a connective tissue disease (specifically and systemic sclerosis). Furthermore, we decided to use DAS28 because, unlike CDAI, it includes the ESR, which represents an objective parameter of the inflammatory phenomenon. Second, the patients included in that study had predominantly inflammatory HRCT patterns, with higher ground glass scores than our patients. This may have influenced the correlation analysis. Third, our study included not only a morphologic (HRCT) but also a functional parameter of lung disease in the correlation analysis. Our study has some limitations. First, although all the $\mathrm{CT}$ scans were of high resolution, they were not performed with the same protocol given the observational and "real life" nature of our study. This fact could have contributed to the heterogeneity of the CT scans, affecting the quality of the study. Furthermore, we were not able to obtain the HRCT scores in all patients, and thus, in a proportion of them, we could only study the correlation between lung function test parameters and joint disease. Second, it would have been ideal to study the correlation between joint and lung disease in patients without treatment, because only in this scenario we can attribute the lung abnormalities to the autoimmune disease and not to the drugs' effect or toxicity. Most of our patients were receiving at least one drug to treat joint inflammation, and so this model scenario was impossible in this cohort. Finally, it is a fact that the evaluation of RA disease activity and ILD course is a dynamic process, and a crosssectional study is not the best design to answer these questions. Our study has an acceptable power to evaluate the correlation between articular and lung involvement in RA ILD patients, and the methods we 
chose to evaluate both components of the disease are standardized instruments. Consequently, we believe that our results are valid and can be generalized. However, we consider that more studies are needed to find a definitive answer to our question.

\section{REFERENCES}

1. Gabriel SE. The epidemiology of rheumatoid arthritis. Rheum Dis Clin North Am. 2001;27:269-81.

2. Tanoue LT. Pulmonary manifestations of rheumatoid arthritis. Clin Chest Med. 1998;19:667-85, viii.

3. Olson AL, Swigris JJ, Sprunger DB, et al. Rheumatoid arthritisinterstitial lung disease-associated mortality. Am J Respir Crit Care Med. 2011:183:372-8.

4. Restrepo JF, del Rincón I, Battafarano DF, et al. Clinical and laboratory factors associated with interstitial lung disease in rheumatoid arthritis. Clin Rheumatol. 2015;34:1529-36.

5. Tanaka N, Kim JS, Newell JD, et al. Rheumatoid arthritis-related lung diseases: CT findings. Radiology. 2004;232:81-91.

6. Assayag D, Elicker BM, Urbania TH, et al. Rheumatoid arthritisassociated interstitial lung disease: Radiologic identification of usual interstitial pneumonia pattern. Radiology. 2014;270:583-8.

7. Assayag D, Lee JS, King TE Jr. Rheumatoid arthritis associated interstitial lung disease: A review. Medicina (B Aires). 2014; 74:158-65.

8. Kim EJ, Elicker BM, Maldonado F, et al. Usual interstitial pneumonia in rheumatoid arthritis-associated interstitial lung disease. Eur Respir J. 2010;35:1322-8.

9. Pérez-Dórame R, Mejía M, Mateos-Toledo H, Rojas-Serrano J. Rheumatoid arthritis-associated interstitial lung disease: Lung inflammation evaluated with high resolution computed tomography scan is correlated to rheumatoid arthritis disease activity. Reumatol Clin. 2015;11:12-6.

10. Perez-Alvarez R, Perez-de-Lis M, Diaz-Lagares C, et al. Interstitial lung disease induced or exacerbated by TNF-targeted therapies: Analysis of 122 cases. Semin Arthritis Rheum. 2011; 41:256-64.

11. Koo BS, Hong S, Kim YJ, Kim YG, Lee CK, Yoo B, et al. Mortality in patients with rheumatoid arthritis-associated interstitial lung disease treated with an anti-tumor necrosis factor agent. Korean J Intern Med. 2015;30:104-9.

12. Wolfe F, Caplan L, Michaud K. Rheumatoid arthritis treatment and the risk of severe interstitial lung disease. Scand J Rheumatol. 2007;36:172-8.

13. Dixon WG, Hyrich KL, Watson KD, et al. Influence of anti-TNF therapy on mortality in patients with rheumatoid arthritis-associated interstitial lung disease: Results from the British society for rheumatology biologics register. Ann Rheum Dis. 2010; 69:1086-91.

14. Paulin F, Doyle TJ, Fletcher EA, Ascherman DP, Rosas IO. Rheumatoid arthritis-associated interstitial lung disease and idiopathic pulmonary fibrosis: Shared mechanistic and phenotypic traits suggest overlapping disease mechanisms. Rev Invest Clin. 2015;67:280-6.

15. Aletaha D, Neogi T, Silman AJ, et al 2010 rheumatoid arthritis classification criteria: An American college of rheumatology/ European league against rheumatism collaborative initiative. Arthritis Rheum. 2010;62:2569-81.
16. Wells G, Becker JC, Teng J, et al. Validation of the 28-joint disease activity score (DAS28) and European league against rheumatism response criteria based on C-reactive protein against disease progression in patients with rheumatoid arthritis, and comparison with the DAS28 based on erythrocyte sedimentation rate. Ann Rheum Dis. 2009;68:954-60.

17. Singh JA, Furst DE, Bharat A, et al 2012 update of the 2008 American college of rheumatology recommendations for the use of disease-modifying Antirheumatic drugs and biologic agents in the treatment of rheumatoid arthritis. Arthritis Care Res (Hoboken). 2012;64:625-39.

18. Miller MR, Crapo R, Hankinson J, et al. General considerations for lung function testing. Eur Respir J. 2005;26:153-61.

19. Raghu G, Collard HR, Egan JJ, et al. An official ATS/ERS/JRS/ ALAT statement: Idiopathic pulmonary fibrosis: Evidence-based guidelines for diagnosis and management. Am J Respir Crit Care Med. 2011;183:788-824

20. Goh NS, Desai SR, Veeraraghavan S, et al. Interstitial lung disease in systemic sclerosis: A simple staging system. Am J Respir Crit Care Med. 2008;177:1248-54.

21. von Elm E, Altman DG, Egger $M$, et al. The strengthening the reporting of observational studies in epidemiology [STROBE] statement: Guidelines for reporting observational studies. Gac Sanit. 2008;22:144-50.

22. Zou YQ, Li YS, Ding XN, Ying ZH. The clinical significance of HRCT in evaluation of patients with rheumatoid arthritis-associated interstitial lung disease: A report from China. Rheumatol Int. 2012:32:669-73.

23. Gochuico BR, Avila NA, Chow CK, et al. Progressive preclinical interstitial lung disease in rheumatoid arthritis. Arch Intern Med. 2008;168:159-66.

24. Fischer A, Solomon JJ, du Bois RM, Deane KD, et al. Lung disease with anti-CCP antibodies but not rheumatoid arthritis or connective Tissue disease. Respir Med. 2012;106:1040-7.

25. Gizinski AM, Mascolo M, Loucks JL, et al. Rheumatoid arthritis (RA)-specific autoantibodies in patients with interstitial lung disease and absence of clinically apparent articular RA. Clin Rheumatol. 2009;28:611-3.

26. Ye W, Fifield MC, Östör AJ. Refractory rheumatoid arthritis and associated interstitial lung disease: Could abatacept be the answer? J Clin Rheumatol. 2017;23:125-6.

27. Wuyts WA, Cavazza A, Rossi G, et al. Differential diagnosis of usual interstitial pneumonia: When is it truly idiopathic? Eur Respir Rev. 2014;23:308-19.

28. Rangel-Moreno J, Hartson L, Navarro C, et al. Inducible bronchus-associated lymphoid tissue (BALT) in patients with pulmonary complications of rheumatoid arthritis. J Clin Invest. 2006; 116:3183-94.

29. Tak PP, Bresnihan B. The pathogenesis and prevention of joint damage in rheumatoid arthritis: Advances from synovial biopsy and tissue analysis. Arthritis Rheum. 2000;43:2619-33.

30. Chatzidionisyou A, Catrina Al. The lung in rheumatoid arthritis, cause or consequence? Curr Opin Rheumatol. 2016;28:76-82.

31. Paulin F, Babini A, Mamani M, Mercado J, Caro F. Practical approach to the evaluation and management of rheumatoid arthritis-interstitial lung disease based on its proven and hypothetical mechanisms. Rev Invest Clin. 2017;69:235-42.

32. Rojas-Serrano J, González-Velásquez E, Mejía M, Sánchez-Rodríguez A, Carrillo G. Interstitial lung disease related to rheumatoid arthritis: Evolution after treatment. Reumatol Clin. 2012;8:68-71.

33. Kazerooni EA, Martinez FJ, Flint A, et al. Thin-section CT obtained at $10-\mathrm{mm}$ increments versus limited three-level thinsection CT for idiopathic pulmonary fibrosis: Correlation with pathologic scoring. AJR Am J Roentgenol. 1997;169:977-83. 